Please note that all letters must be typed. Priority will be given to those that are less than $\mathbf{5 0 0}$ words long. All authors must sign the letter, which may be shortened or edited for reasons of space or clarity. All letters received are acknowledged.

\section{Piercing issue}

Sir, - A teenage patient who attended my department for a fixed appliance to be fitted was wearing a small jewel bonded onto one of her incisors. It turned out that this had been put in place by her hairdresser and my nurses told me that it is quite common for such decorations to be applied by beauticians, 'piercing parlours' and tattooists.

Leaving aside any legal aspects I am particularly concerned as an orthodontist that any attachment left permanently in place be it a decoration of this type or an orthodontic bracket - will inevitably provide an area where plaque can accumulate and can only be justified in a patient who has a good standard of oral hygiene at the outset and is able to demonstrate continuing good plaque control while the attachment is in place.

I would be interested to know whether any members of the profession have seen examples of decalcification or cavitation from 'jewels' bonded onto enamel and what the legal position of unqualified people who provide such attachments is likely to be.

\section{J. D. Muir}

\section{Stoke-on-Trent}

Linda Wallace from the BDA's Health and Science Policy Group says:

There is a very small risk of damage to the tooth enamel; there is also a risk that the jewellery might be accidentally detached and inhaled. It is recommended that such jewellery be placed by a dentist, who is in the best position to explain the risks prior to provision and to give oral hygiene advice. Dentists will, of course, also be aware of the appropriate infection control protocols and of the importance of not placing tooth jewellery inappropriately (on teeth that require treatment or on restorations that could be damaged).

Regarding the legal position, the General Dental Council distinguishes between jewellery that is simply stuck on and that which is bonded by an acid etch. The Council's view is that the application of tooth jewellery to a tooth by an unqualified person, provided that it involves no preparation of the tooth, cannot be described as the practice of dentistry within the meaning of the Dentists Act 1984 and, therefore, is not a criminal offence under that legislation.

Should, however, the application of tooth jewellery involve the preparation of the tooth by such means as the etching of the tooth prior to application, this clearly constitutes the practice of dentistry and, as such, is an illegal practice by an unqualified practitioner, which could result in prosecution.

\section{Outreach clinics}

Sir,- I would like to congratulate O'Brien et al for their recent paper (BDJ 2001; 191 : 203-207).

Determining the comparative costs and effectiveness of providing specialist treatment in more accessible settings is an area of increasing interest. The data is high quality (evidence level 1b) and therefore strong evidence for health service policy decisionmaking. However, their conclusions should be challenged. The evidence presented shows there are clear advantages of providing services in outreach clinics.

The study explored several factors. Unfortunately, the clinical outcomes were restricted to first visit attendance rates and the decision to provide treatment or not following this visit. The unsurprising finding that both locations achieved the same outcomes at this limited end-point puts the focus on other factors to determine the relative advantage of the different service locations. These factors were patient perception and provider costs. In both these factors their evidence shows clear advantages in favour of outreach clinics.

For patients, the time spent travelling to and in the outreach clinics was significantly less compared to the main base unit and presumably also incurred less patient costs (financial and opportunity). Satisfaction with the outreach clinics was also higher.

The main challenge to this positive perception of outreach clinics was that most patients, from both groups, stated they would be happy to see a specialist in another setting. This is surely a reflection of patients' demand for specialist treatment not dissatisfaction with outreach clinic location.

Overall, the time, cost and satisfaction advantages to patients attending outreach clinics are higher than those attending the main base unit. Over a lengthy period of treatment, these advantages could lead to better rates of attendance, compliances and treatment completion.

For providers, the cost per patient visit in the main base is about $8.5 \%$ more than the outreach clinics. These figures include travel and opportunity costs incurred by specialists. This is a cost saving in the region of $7.8 \%$ per patient visit. Outreach clinics give a clear provider cost advantage.

If the patient advantages outlined above do lead to lower rates of failed appointments and higher rates of successful treatment outcomes, the cost savings to providers and overall cost-effectiveness becomes greater still in favour of outreach clinics.

In conclusion, this paper provides evidence that there are marked advantages to both patients and provider costs. The study is limited by the treatment outcome measure selected.

To further aid policy decision-making, more evidence is required to determine whether these advantages lead to better attendance and treatment outcome rates over the period required for completing specialist orthodontic treatment.

It would be interesting to see the results of this study over, say, a three year period, particularly in relation to outcomes addressing health inequalities.

\section{A. Sprod}

Bristol

Sir, - Surely this paper on outreach clinics has a major flaw in the poor choice of clinic sites. The first site appears to be 500 metres - not much more than the length of a hospital corridor - away from the main clinic. The second is $2 \mathrm{~km}$ and the third a mere $15 \mathrm{~km}$ from the main base! These cannot be 'outreach clinics', they are 'near-reach clinics.'

Several benefits are claimed for outreach clinics in general; increased interaction of primary and secondary care providers; reduction of waiting; but the main parameter is increased consumer accessibility in the form of reduced travelling time and cost for patient.

The choice of sites in this paper gave no hope for this parameter to show any benefit. The question of subconscious bias must arise and perhaps it would have been better for the sites to be chosen by an independent researcher with guidelines on what an outreach clinic is.

I note also that despite making a point of the variation in distance of clinics from the main base, the authors have failed to follow this up and distinguish differences in accessibility and costs between the outreach clinics.

'Outreach' clinics are designed to help 'outlying' populations. The critical factors in choosing sites are the potential patient population and the distance from main site. I have been involved in provision of military outreach clinics for 15 years.

I cannot offer the 'scientific power' of a randomised control trial, but I can offer commonsense and personal communication to say that, if sited correctly, outreach clinics are very advantageous. Did anyone honestly believe that a distance of 500 metres would significantly improve consumer accessibility? If so, then this paper was not wasted?

P. T. R. Ollivere

Eastbourne 
Co-author Kevin O'Brien responds: We would like to thank Dr Sprod for his constructive and helpful comments on our paper. We feel that we interpreted the data in a balanced way and while there appeared to be some advantages in outreach clinics, these were not great. Other important factors such as the rate of failed appointments and outcome of care were no different between the two settings.

When we consider our choice of outcome of new patient consultations, this was the simple aim of our investigation. We explained the rationale for this choice in the introduction of the paper. As a result, this study should be considered to be a first step in studies into the effectiveness of outreach clinics. It is certainly a quantum leap to extrapolate the results of our study to the provision of lengthy courses of orthodontic treatment in outreach clinics. We agree that this should be subject of a much more complex and costly investigation.

As regards the comments of Dr Ollivere, he does raise some important points about the siting of the outreach clinics in our study. However, more careful reading of our paper would reveal that we fully discussed these issues.

Importantly, we stated that the findings were only relevant to large conurbations where in addition to the distance travelled we need to consider the time that is involved in taking public transport on congested roads and/or difficulties in parking. For example, the time needed to travel on public transport from our most distant clinic is 60 minutes involving two changes of bus. While Dr Ollivere feels that this is a short distance, I am sure that most of our less advantaged patients do not feel the same.

As regards his comments about our selection of clinics, as we stated in the paper, we selected clinics that were relevant to our catchment area. We did look for differences between the clinics, but we did not find any. As a result, in order to keep the paper brief and to the point, we omitted this data.

Finally, we wonder if Dr Ollivere would have written his letter if the findings of our scientific randomised controlled trial into outreach clinics sited in inner city Manchester had agreed with his personal opinion on the provision of outreach in the military (two situations that are hardly comparable!).

Sir,- I read with interest the trial reported by O'Brien and colleagues evaluating specialist outreach clinics for orthodontic consultation (BDJ 2001; 191: 203-7).

The authors are to be congratulated on this thoughtful work. Evaluative research of this type is needed not only to appraise the effectiveness of interventions but also the way in which care is delivered.

One aspect of the study design that may have influenced the findings was the proximity of the three outreach clinics to the main base $(500 \mathrm{~m}, 2 \mathrm{~km}$. and $15 \mathrm{~km})$. An indication of the relationship between these distances and the accessibility of care may be helpful to providers.

It could be that an outreach centre $15 \mathrm{~km}$ from a main base is more easily justified on grounds of enhanced accessibility than one 500 m away.

\section{P. G. Robinson}

\section{Senior Clinical Lecturer, GKT Dental Institute \\ London}

\section{Questions, questions...}

Sir, - This year, so far, I have received three questionnaires with a request to complete them. One on linings (what do I use to line cavities, and in what situations); one on my cross-infection control practice; and the third on how I am ageing (what complaints do I suffer from, what is my weight, how far has my hairline receded?)

I have dutifully filled them all in - sometimes after a polite reminder — and then heard absolutely nothing.

What I would like the sender of such questionnaires to do, is send me a copy of the final paper. I want to know if my procedures are acceptable, whether I am using the most appropriate materials, and whether I am ageing well or badly. If the researchers did offer to send the results of their work, I believe they would receive more replies, and often help to educate the recipient of their surveys.

\section{R. Yewe-Dyer Alton}

\section{'Drop in' dentistry}

Sir-_ Congratulations to Y. M. Dailey and M. Martin on their thought-provoking paper (BDJ 2001; 191:391-393.

The authors concentrated on part of an increasing problem, which is 'drop in dentistry'. While I would agree with their limited conclusions, viz a vie that 'antibiotics are being inappropriately prescribed,' I feel that the very nature of 'drop in dentistry' itself has more to do with the problem.

'Drop in dentistry' is actually 'lowest common denominator dentistry.' Because people now know that there is an emergency facility available, there is no incentive to get or keep an appointment with a 'proper' dentist.

Surely it would have been better for the health authorities to have simply purchased time from GDPs to see unregistered emergencies on a daily basis, in their own practices. Of course, this would not help to build empires or edifices, and the photo opportunities would be diminished, but as a conso- lation, the patients would receive a better quality of overall care, and it would cost a fraction of what it does now.

\section{G. Allen}

\section{St. Helens}

Sir,- - I read with interest Daily and Martin's paper with regard to 'inappropriate use of antibiotics for emergency dental treatment.' I am a GDP of over 30 years experience, I work in Cardiff and can assure you that the same results would be found here in emergency clinics as in Cheshire.

I have lost count of the number of patients that have attended my surgery in severe pain having already been to a weekend emergency clinic for so called 'emergency care'.

In most cases they have been 'briefly' examined and then given a prescription for antibiotics. No attempt has been made to carry out any clinical treatment to the tooth. They have then been told to visit their own GDP as soon as possible. In many cases simple root canal drainage or dressing would have done more for the patient than any antibiotic.

It has made me very disappointed in members of my profession. To be able to relieve a patient's painful symptoms is the first and foremost requirement of any dental surgeon, and when colleagues fail to do this to the best of their ability, they are betraying the Hippocratic oath and themselves. Also the health authorities that pay out large sums of money for emergency services are being duped.

The last question to be answered is "Why do some dentists have this attitude?' I was never taught to treat dental pain with antibiotics alone, unless there was no other choice. I suspect its just down to a poor uncaring attitude in most cases - a great shame.

\section{P. A. James \\ Cardiff}

Please send your letters to:

$$
\begin{aligned}
& \text { The Editor } \\
& \text { British Dental Journal } \\
& 64 \text { Wimpole Street } \\
& \text { London } \\
& \text { W1M 8AL }
\end{aligned}
$$

